\title{
Compensation Improvement of DPSK Signal With Nonlinear Phase Noise
}

\author{
Keang-Po Ho, Member, IEEE
}

\begin{abstract}
When nonlinear phase noise is compensated by the received intensity, simple formulas are derived for the error probability of differential phase-shift keying signals. Simulation is conducted to verify the error probability. The tolerance of nonlinear phase noise is doubled by the compensator, allowing doubling of the transmission distance if nonlinear phase noise is the dominant impairment.
\end{abstract}

Index Terms-Differential phase-shift keying (DPSK), fiber nonlinearities, nonlinear phase noise, phase modulation.

\section{INTRODUCTION}

$\mathbf{N}$ ONLINEAR phase noise, often called the Gordon-Mollenauer effect [1], is induced by the interaction of the fiber Kerr effect and optical amplifier noise. Both phase-shift keying (PSK) and differential phase-shift keying (DPSK) signals are degraded by nonlinear phase noise [1]-[6]. DPSK signaling has renewed interests recently for both long-haul and spectral efficiency transmission systems [7]-[11]. Correlated with the nonlinear phase noise, the received intensity can be used to compensate the nonlinear phase noise [12]-[15] to about half its standard deviation [12]-[14].

Recently, Kim and Gnauck [5] show experimentally and the author [16], [17] shows theoretically that the nonlinear phase noise is not Gaussian distributed. The variance or $Q$ factor [1], [4], [12]-[14] is not sufficient to characterize the system performance. Using the probability density function (pdf) from [16], this letter evaluates the error probability for DPSK systems with and without compensation.

This letter assumes that both the nonlinear phase noise without compensation and the residual nonlinear phase noise after compensation are independent of the phase component of the amplifier noise. Using the Fourier series expansion of the pdf of the phase noise [18]-[20], closed-form formulas are derived to calculate the error probability of DPSK signals with either nonlinear phase noise or residual nonlinear phase noise. The signal-to-noise ratio (SNR) penalty is also calculated for an error probability of $10^{-9}$. Monte Carlo simulation is conducted to verify the formulas.

Manuscript received March 11, 2003; revised May 19, 2003.

The author is with the Graduate Institute of Communication Engineering, National Taiwan University, Taipei 106, Taiwan, R.O.C. (e-mail: kpho@cc.ee.ntu.edu.tw).

Digital Object Identifier 10.1109/LPT.2003.816703

\section{Closed-Form ERror ProbabiLITy}

With uncompensated nonlinear phase noise, a DPSK signal is demodulated using the differential phase of

$$
\begin{aligned}
\Delta \Phi_{r}= & \Phi_{r}(t)-\Phi_{r}(t-T) \\
= & \theta(t)+\Theta_{n}(t)-\Phi_{\mathrm{NL}}(t) \\
& -\theta(t-T)-\Theta_{n}(t-T)+\Phi_{\mathrm{NL}}(t-T)
\end{aligned}
$$

where $\Phi_{r}(\cdot), \theta(\cdot), \Theta_{n}(\cdot)$, and $\Phi_{\mathrm{NL}}(\cdot)$ are the received phase, the transmitted phase, the phase of amplifier noise, and the nonlinear phase noise as a function of time, and $T$ is the symbol time. The phases at $t$ and $t-T$ are independent identically distributed random variables. Error probability is calculated using the pdf of $\Delta \Phi_{r}$. The pdf of the phase of amplifier noise can be found in [6] and [18]-[20]. The characteristic function of the nonlinear phase noise can be found in [16] and [17].

Having a period of $2 \pi$, the pdf of the phase of amplifier noise of $\Theta_{n}$ has a Fourier series of [18]-[20]

$$
p_{\Theta_{n}}(\theta)=\frac{1}{2 \pi}+\frac{1}{\pi} \sum_{m=1}^{\infty} c_{m} \cos (m \theta)
$$

with coefficients of

$$
\begin{aligned}
c_{m} & =\frac{\Gamma\left(\frac{m}{2}+1\right) \rho_{s}^{m / 2}}{m !}{ }_{1} F_{1}\left(\frac{m}{2} ; m+1 ;-\rho_{s}\right) \\
& =\frac{\sqrt{\pi \rho_{s}}}{2} e^{-\rho_{s} / 2}\left[I_{(m-1) / 2}\left(\frac{\rho_{s}}{2}\right)+I_{(m+1) / 2}\left(\frac{\rho_{s}}{2}\right)\right]
\end{aligned}
$$

where $\rho_{s}$ is the SNR, $\Gamma(\cdot)$ is the Gamma function, ${ }_{1} F_{1}(a ; b ; \cdot)$ is the confluent hypergeometric function of the first kind, and $I_{\nu}(\cdot)$ is the $\nu$ th order modified Bessel function of the first kind. Using a series similar to (2), the error probability is found for DPSK signals with noisy reference [19], phase error [20], or laser phase noise [21].

Assume that the signal phases at $t$ and $t-T$ are the same with $\theta(t)=\theta(t-T)$. If the phase of amplifier noise of $\Theta_{n}$ is independent of the nonlinear phase noise of $\Phi_{\mathrm{NL}}$, the pdf of the differential phase is

$$
p_{\Delta \Phi_{r}}(\theta)=\frac{1}{2 \pi}+\frac{1}{\pi} \sum_{m=1}^{\infty} c_{m}^{2}\left|\Psi_{\Phi_{\mathrm{NL}}}(j m)\right|^{2} \cos (m \theta)
$$

where $\Psi_{\Phi_{\mathrm{NL}}}(\cdot)$ is the characteristic function of the nonlinear phase noise from [16]. In (4), the coefficients of $c_{m}^{2}$ and $\left|\Psi_{\Phi_{\mathrm{NL}}}(j m)\right|^{2}$ correspond to the term of $\Theta_{n}(t)-\Theta_{n}(t-T)$ and $\Phi_{\mathrm{NL}}(t)-\Phi_{\mathrm{NL}}(t-T)$, respectively, in the differential phase of (1). In the pdf (4), the Fourier coefficients of the addition (or subtraction) of the differential phase of (1) give the multiplication of the corresponding Fourier coefficients. 
Based on interferometer, the direct-detection DPSK receiver provides a decision variable proportional to $\cos \left(\Delta \Phi_{r}\right)$ [7], [9]-[11]. The receiver makes the decision based on whether $\cos \left(\Delta \Phi_{r}\right)$ is positive or negative that is equivalent to whether $\Delta \Phi_{r}$ is within or without the angle of $\pm \pi / 2$. The error probability for DPSK signal is

$$
p_{e, \mathrm{NL}}=1-\int_{-\pi / 2}^{\pi / 2} p_{\Delta \Phi_{r}}(\theta) d \theta
$$

or

$$
p_{e, \mathrm{NL}}=\frac{1}{2}-\frac{1}{\pi} \sum_{m=1}^{\infty} c_{m}^{2}\left|\Psi_{\Phi_{\mathrm{NL}}}(j m)\right|^{2} \frac{2 \sin \left(\frac{m \pi}{2}\right)}{m} .
$$

Because $\sin (m \pi / 2)=0$ if $m$ is an even number, we have

$$
\begin{aligned}
p_{e, \mathrm{NL}}=\frac{1}{2}-\frac{\rho_{s} e^{-\rho_{s}}}{2} \sum_{k=0}^{\infty} \frac{(-1)^{k}}{2 k+1} & {\left[I_{k}\left(\frac{\rho_{s}}{2}\right)+I_{k+1}\left(\frac{\rho_{s}}{2}\right)\right]^{2} } \\
& \times\left|\Psi_{\Phi_{\mathrm{NL}}}[(2 k+1) j]\right|^{2}
\end{aligned}
$$

Efficiently calculated, the error probability of (7) gives about the same results as that in [6]. With a simple closed-form expression of (7), the more complicated characteristic functions from [16] can be used in the calculation.

As shown in [14], the differential received intensity of $\Delta P=$ $P(t)-P(t-T)$ can be used to compensate for the differential nonlinear phase noise of $\Phi_{\mathrm{NL}}(t)-\Phi_{\mathrm{NL}}(t-T)$ using the same optimal scale factor as that in [14]. Similar to the procedure from (4) to (7), the error probability with nonlinear phase noise compensation is

$$
\begin{aligned}
& p_{e, \mathrm{RES}}=\frac{1}{2}-\frac{\rho_{s} e^{-\rho_{s}}}{2} \sum_{k=0}^{\infty} \frac{(-1)^{k}}{2 k+1} {\left[I_{k}\left(\frac{\rho_{s}}{2}\right)+I_{k+1}\left(\frac{\rho_{s}}{2}\right)\right]^{2} } \\
& \times\left|\Psi_{\Phi_{\mathrm{RES}}}[(2 k+1) j]\right|^{2}
\end{aligned}
$$

where $\Psi_{\Phi_{\mathrm{RES}}}(\cdot)$ from [16] is the characteristic function of the residual nonlinear phase noise after compensation.

The formulas of (7) and (8) are similar to that of [19] with noisy reference and that of [21] with laser phase noise. If the nonlinear phase noise is assumed to be Gaussian distributed and independent of the phase of amplifier noise, the error probability can be calculated by [21].

\section{NUMERICAL RESULTS}

Fig. 1 shows the error probability of DPSK signal as a function of SNR $\rho_{s}$. The error probability without and with compensation is calculated by the formulas of (7) and (8), respectively. The system in Fig. 1 has 32 identical fiber spans. In Fig. 1, the error probability with different values of mean nonlinear phase shift $\left\langle\Phi_{\mathrm{NL}}\right\rangle$ is shown for comparison. Without nonlinear phase noise, the error probability is $\exp \left(-\rho_{s}\right) / 2$ [6], [19], [20]. When the nonlinear phase noise is compensated using the received intensity with the optimal scale factor from [14] or the approximated scale factor from [13], the standard deviation of the nonlinear phase noise is reduced by a factor of about two [12]-[14], [16]. Comparing Fig. 1(a) with Fig. 1(b), the error probability

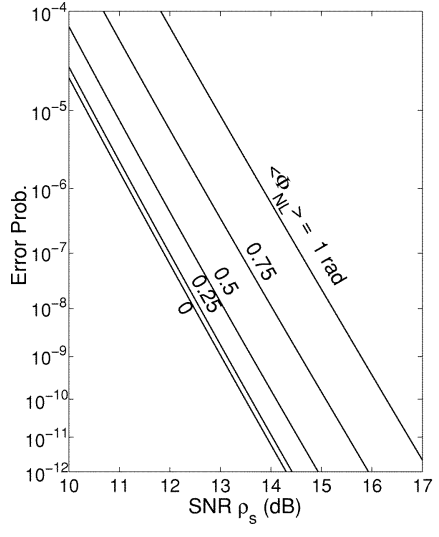

(a)

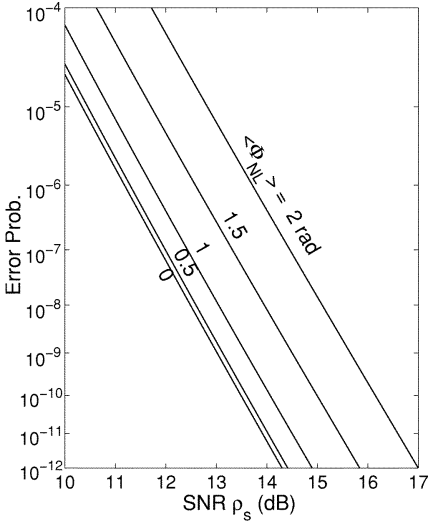

(b)
Fig. 1. Error probability of DPSK signal with nonlinear phase noise (a) without and (b) with compensation

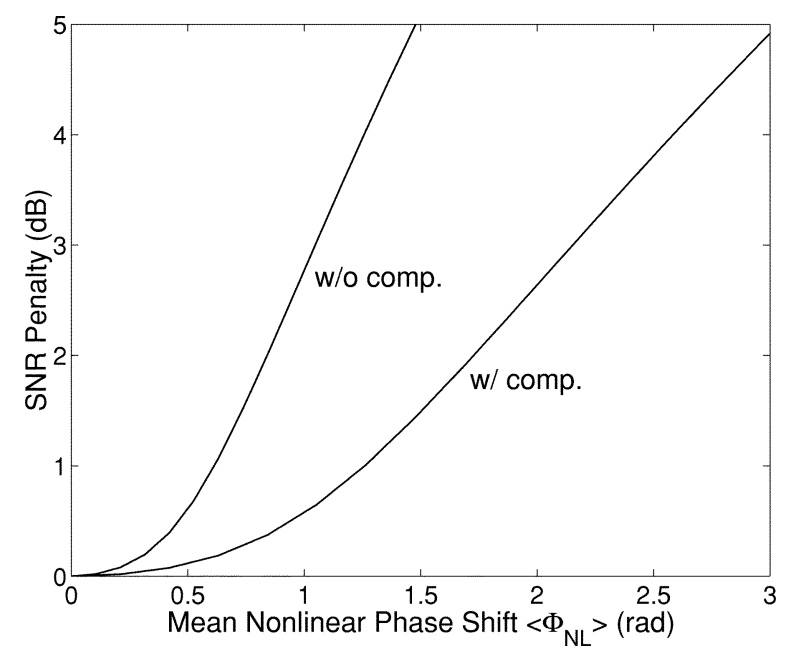

Fig. 2. SNR penalty of an error probability of $10^{-9}$ as a function of mean nonlinear phase shift of $\left\langle\Phi_{\mathrm{NL}}\right\rangle$.

of DPSK signal without compensation and having a mean nonlinear phase shift of $\left\langle\Phi_{\mathrm{NL}}\right\rangle$ is slightly larger than that with compensation but having twice the mean nonlinear phase shift of $2\left\langle\Phi_{\mathrm{NL}}\right\rangle$.

Without compensation, the error probability in Fig. 1(a) is similar to that in [6] but using the more complicated pdf from [16] instead of the asymptotic pdf from [17]. From [17], the tail probability of the pdf with 32 fiber spans is larger than that with infinitely many fiber spans and the error probability of Fig. 1(a) is slightly larger than that from [6].

Fig. 2 shows the SNR penalty for an error probability of $10^{-9}$ as a function of mean nonlinear phase shift of $\left\langle\Phi_{\mathrm{NL}}\right\rangle$ for the same system of Fig. 1. Mean nonlinear phase shifts of $\left\langle\Phi_{\mathrm{NL}}\right\rangle=0.62$ and $1.27 \mathrm{rad}$ give a SNR penalty of $1 \mathrm{~dB}$ without and with compensation, respectively. For the same power penalty, the DPSK system with compensation can tolerate a mean nonlinear phase shift slightly larger than twice of that of the system without compensation, confirming the same results from [12]-[14], [16], and Fig. 1.

Similar to [6], the derivations from (4) to (8) assume that the phase of amplifier noise $\Theta_{n}$ is independent to the nonlinear phase noise of $\Phi_{\mathrm{NL}}$ or the residual nonlinear phase noise of $\Phi_{\text {RES }}$. It is obvious that the phase of amplifier noise is correlated 


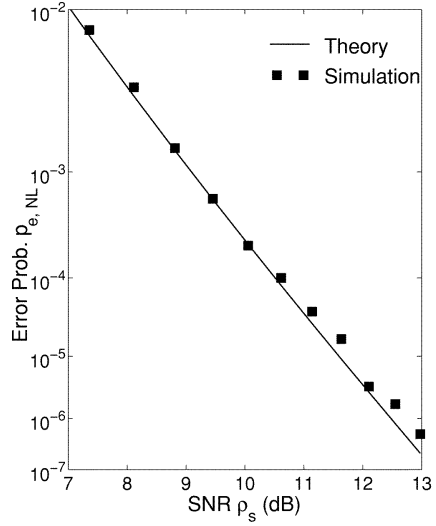

(a)

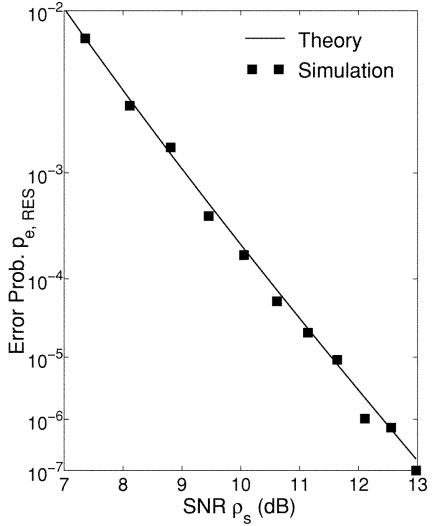

(b)
Fig. 3. Simulated error probability of DPSK signal with nonlinear phase noise (a) without and (b) with compensation.

to neither the nonlinear phase noise of $\Phi_{\mathrm{NL}}$ nor the residual nonlinear phase noise of $\Phi_{\mathrm{RES}}$. However, as non-Gaussian random variables, they may be weakly dependent [6].

Fig. 3 show little difference between the simulated and theoretical error probability. There is a $0.2-\mathrm{dB}$ SNR difference for the same error probability. Fig. 3(a) and (b) has mean nonlinear phase shifts of $\left\langle\Phi_{\mathrm{NL}}\right\rangle=1 / \sqrt{2}$ and $\sqrt{2}$, without and with compensation, respectively, for an SNR penalty around $1 \mathrm{~dB}$. The variance of the phase of amplifier noise is about 1.5 times the variance of the nonlinear phase noise or the residual nonlinear phase noise [1], [14].

The simulation of Fig. 3 is conducted similar to [6] and [14] but for DPSK signals. Equivalently speaking, the signal distribution for DPSK signal (see [14, Fig. 2] for PSK signal) is found and the error probability is calculated by counting the number of points outside the decision region. We count at least ten errors to ensure a good confident interval [22, Fig. 2].

The SNR of Figs. 1 and 3 is defined the same as that in [6] and [17]-[21]. When optical SNR (OSNR) is measured using an optical spectrum analyzer with a bandwidth of $\mathrm{BW}_{\mathrm{opt}}$, the SNR is related to OSNR by $\rho_{s}=2 \mathrm{OSNR} \times \mathrm{BW}_{\text {opt }} / R_{b}$ where $R_{b}$ is the data rate of the signal and the factor of two assumes a polarization-insensitive optical spectrum analyzer.

\section{CONCLUSION}

Closed-form formulas are derived for the error probability of DPSK signals contaminated by nonlinear phase noise with and without compensation using the received intensity. The error probability is derived based on the assumption that the phase of amplifier noise is independent of both the nonlinear phase noise without compensation and the residual nonlinear phase noise after compensation. Simulation shows that the error probability formulas are very accurate. For the same SNR penalty, the mean nonlinear phase shift is double, doubling the transmission distance if nonlinear phase noise is the dominant impairment.

\section{REFERENCES}

[1] J. P. Gordon and L. F. Mollenauer, "Phase noise in photonic communications systems using linear amplifiers," Opt. Lett., vol. 15, pp. 1351-1353, 1990.

[2] S. Ryu, "Signal linewidth broadening due to nonlinear Kerr effect in long-haul coherent systems using cascaded optical amplifiers," J. Lightwave Technol., vol. 10, pp. 1450-1457, Oct. 1992.

[3] S. Saito, M. Aiki, and T. Ito, "System performance of coherent transmission over cascaded in-line fiber amplifiers," J. Lightwave Technol., vol. 11, pp. 331-342, Feb. 1993.

[4] C. J. McKinstrie and C. Xie, "Phase jitter in single-channel soliton systems with constant dispersion," IEEE J. Select. Topics Quantum Electron., vol. 8, pp. 616-625, May/June 2002.

[5] H. Kim and A. H. Gnauck, "Experimental investigation of the performance limitation of DPSK systems due to nonlinear phase noise," IEEE Photon. Technol. Lett., vol. 15, pp. 320-322, Feb. 2003.

[6] K.-P. Ho, "Performance degraduation of phase-modulated systems with nonlinear phase noise," IEEE Photon. Technol. Lett., vol. 15, Sept. 2003.

[7] A. H. Gnauck, G. Raybon, S. Chandrasekhar, J. Leuthold, C. Doerr, L. Stulz, A. Agrawal, S. Banerjee, D. Grosz, S. Hunsche, A. Kung, A. Marhelyuk, D. Maymar, M. Movassaghi, X. Liu, C. Xu, X. Wei, and D. M. Gill, " $2.5 \mathrm{tb} / \mathrm{s}(64 \times 42.7 \mathrm{~Gb} / \mathrm{s})$ transmission over $40 \times 100$ km NZDSF using RZ-DPSK format and all-raman-amplified spans," in Proc. OFC, 2002, Postdeadline Paper FC2.

[8] R. Griffin, R. Johnstone, R. Walker, J. Hall, S. Wadsworth, K. Berry, A. Carter, M. Wale, P. Jerram, and N. Parsons, " $10 \mathrm{~Gb} / \mathrm{s}$ optical differential quadrature phase shift key (DQPSK) transmission using GaAs/AlGaAs integration," in Proc. OFC, 2002, Postdeadline Paper FD6.

[9] Y. Miyamoto, H. Masuda, A. Hirano, S. Kuwahara, Y. Kisaka, H. Kawakami, M. Tomizawa, Y. Tada, and S. Aozasa, "S-band WDM coherent transmission of $40 \times 43-G b i t / s$ CS-RZ DPSK signals over 400 km DSF using hybrid GS-TDFAs/Raman amplifiers," Electron. Lett., vol. 38, pp. 1569-1570, 2002.

[10] H. Bissessur, G. Charlet, E. Gohin, C. Simonneau, L. Pierre, and W. Idler, "1.6 Tbit/s $(40 \times 40 \mathrm{Gbit} / \mathrm{s})$ DPSK transmission over $3 \times 100 \mathrm{~km}$ of TeraLight fiber with direct detection," Electron. Lett, vol. 39, pp. 192-193, 2003.

[11] A. Gnauck, G. Raybon, S. Chandrasekhar, J. Leuthold, C. Doerr, L. Stulz, and E. Burrows, "25 40-Gb/s copolarized DPSK transmission over 12 100-km NZDF with 50-Ghz channel spacing," IEEE Photon. Technol. Lett., vol. 15, pp. 467-469, Mar. 2003.

[12] X. Liu, X. Wei, R. E. Slusher, and C. J. McKinstrie, "Improving transmission performance in differential phase-shift-keyed systems by use of lumped nonlinear phase-shift compensation," Opt. Lett., vol. 27, pp. 1616-1618, 2002.

[13] C. $\mathrm{Xu}$ and $\mathrm{X}$. Liu, "Postnonlinearity compensation with data-driven phase modulators in phase-shift keying transmission," Opt. Lett., vol. 27, pp. 1619-1621, 2002.

[14] K.-P. Ho and J. M. Kahn, "Electronic compensation technique to mitigate nonlinear phase noise," J. Lightwave Technol., submitted for publication.

[15] C. Xu, L. F. Mollenauer, and X. Liu, "Compensation of nonlinear selfphase modulation with phase modulators," Electron. Lett., vol. 38, pp. 1578-1579, 2002.

[16] K.-P. Ho, "Probability density of nonlinear phase noise," J. Opt. Soc. Amer. B, vol. 20, no. 9, Sept. 2003, to be published.

[17] — "Asymptotic probability density of nonlinear phase noise," Opt. Lett., vol. 28, no. 15, Aug. 2003, to be published.

[18] D. Middleton, An Introduction to Statistical Comunication Theory. New York: McGraw-Hill, 1960, pp. 414-419.

[19] P. C. Jain, "Error probabilities in binary angle modulation," IEEE Trans. Inform. Theory, vol. IT-20, pp. 36-42, 1974.

[20] N. M. Blachman, "The effect of phase error on DPSK error probability," IEEE Trans. Commun., vol. COM-29, pp. 364-465, 1981.

[21] G. Nicholson, "Probability of error for optical heterodyne DPSK system with quantum phase noise," Electron. Lett., vol. 20, pp. 1005-1007, 1984.

[22] M. C. Jeruchim, "Techniques for estimating the bit error rate in the simulation of digital communication systems," IEEE J. Select. Areas Commun., vol. SAC-2, pp. 153-170, 1984. 